Supporting information for

\title{
Ultra-high Efficient FRET Ratiometric Fluorescence Biosensor for Visual Detection of Alkaline Phosphatase Activity and Its Inhibitor
}

Yuanjin Zhan ${ }^{1,3}$, Shuangting Yang ${ }^{2}$, Lifen Chen², Yanbo Zeng ${ }^{2}$, Lei Li², Zhenyu Lin ${ }^{3}$, Longhua $\mathrm{Guo}^{2 *}$, and Wei $\mathrm{Xu}^{1 *}$

${ }^{1}$ College of Pharmacy, Fujian University of Traditional Chinese Medicine, Fuzhou, Fujian, 350002, China.

${ }^{2}$ Jiaxing Key Laboratory of Molecular Recognition and Sensing; College of Biological, Chemical Sciences and Engineering, Jiaxing University, Jiaxing, Zhejiang 314001, China.

${ }^{3}$ MOE Key Laboratory for Analytical Science of Food Safety and Biology; Fujian Provincial Key Laboratory of Analysis and Detection Technology for Food Safety; College of Chemistry, Fuzhou University, Fuzhou, 350116, China

*Corresponding Authors: guolh@zjxu.edu.cn, guolh@,fzu.edu.cn (Longhua Guo) 2000017@,fjtcm.edu.cn (Wei Xu)

Number of pages: 10

Number of figures: 4

Number of tables: 2 


\section{Table of Contents}

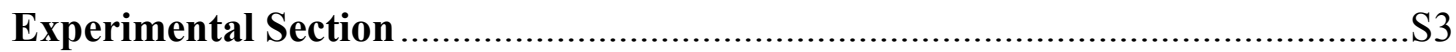

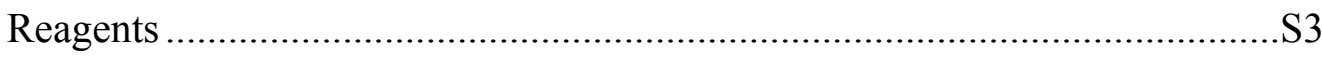

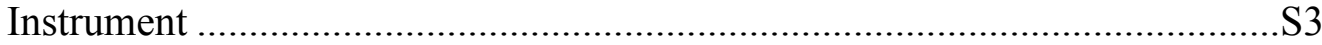

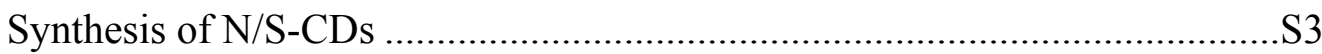

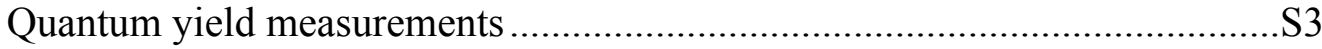

Supplementary characterization, table and figures …….................................... 4

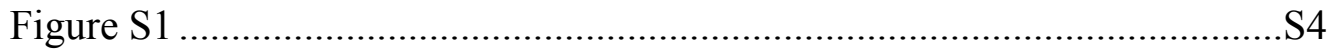

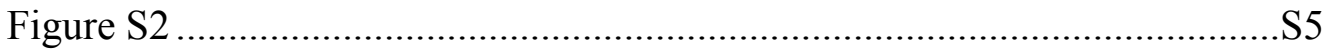

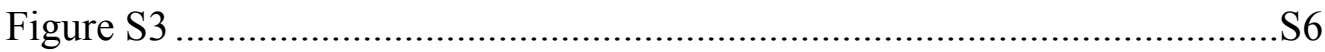

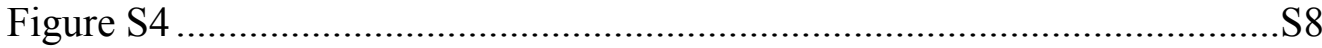

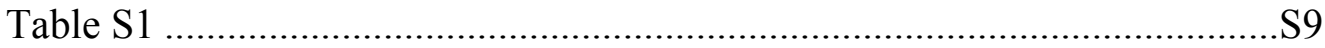

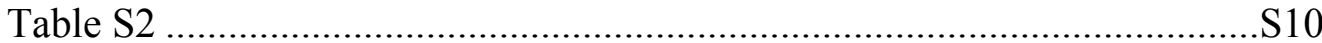




\section{Experimental section}

1.1 Reagents Sodium pyrophosphate ((PPi), copper chloride $\left(\mathrm{CuCl}_{2}\right)$, citric acid, cysteamine hydrochloride, and sodium vanadate $\left(\mathrm{Na}_{3} \mathrm{VO}_{4}\right)$ were purchased from Shanghai Sinopharm Chemical Reagent Co., Ltd. o-phenylenediamine ( $o$ PD) was purchased from Shanghai Aladdin Reagent Co., Ltd. ALP was purchased from Sigma-Aldrich Reagent Co., Ltd (USA). All reagents were of analytical grade and the water was purified by ultra-pure system (Millipore Milli-Q, $18 \mathrm{M} \bullet \mathrm{cm}$ ).

1.2 Instrument Fluorescence spectrometer (F-4600, Hitachi, Japan), UV-Vis NIR spectrometer (UV-2310, Shimadzu, Japan), high resolution transmission electron microscope (HRTEM, TECNAI G2 F20, FEI, USA), transient and steady-state fluorescence spectroscopy system (FL-FS 920 TCSPC, Edinburgh Instruments, UK), Fourier transform infrared spectrum FT-IR system (Nicolet iS50, Thermo Fisher Scientific, USA), digital SLR camera (EOS 600D, Canon, Japan).

1.3 Synthesis of N/S-CDs. Dissolve $2.0 \mathrm{~g}$ of citric acid monohydrate $(9.5 \mathrm{mM})$ and $1.0 \mathrm{~g}$ of cysteamine hydrochloride $(8.8 \mathrm{mM})$ in $5.0 \mathrm{~mL}$ of water. After the ultrasonic is completely dissolved and homogenized, the solution is placed in an oven at $80{ }^{\circ} \mathrm{C}$ for $12 \mathrm{~h}$, the water was evaporated to form a homogenate, and it was transferred to a high-temperature and high-pressure reactor lined with polytetrafluoroethylene and heated at $200{ }^{\circ} \mathrm{C}$ for $3.5 \mathrm{~h}$. After being naturally cooled to room temperature, the solid product was washed with water and freeze-dried or dried at $65{ }^{\circ} \mathrm{C}$ to obtain brown powder, namely, N/S-CDs.

1.4 Quantum yield measurements. The relative quantum yields of the products were measured via compare the integrated emission intensity $\left(\lambda_{\mathrm{ex}}=360 \mathrm{~nm}\right)$ and absorbance at $360 \mathrm{~nm}$ with those of quinine sulfate (in $0.5 \mathrm{M} \mathrm{H}_{2} \mathrm{SO}_{4}, \Phi=0.54$ ) using a single point of concentration. To minimize the reabsorption effect, the absorbance of all solutions at $360 \mathrm{~nm}$ was less than 0.10 .

$$
\Phi_{x}=\Phi_{s t}\left(I_{x} / I_{s t}\right)\left(\eta_{x} / \eta_{s t}\right)^{2}\left(A_{s t} / A_{x}\right)
$$

Where $\Phi$ is the relative quantum yield, $I$ is the measured integrated emission intensity, $\eta$ is the refractive index of the solvent, and $A$ is the optical density. The subscript "st" refers to quinine sulfate dissolved in $0.5 \mathrm{M} \mathrm{H}_{2} \mathrm{SO}_{4}$ with absolute quantum yield (0.54), and "x" for the sample. In order to minimize re-absorption effects, absorption in the $1.0 \mathrm{~cm}$ fluorescence cuvette was kept below 0.10 at the excitation wavelength $(360 \mathrm{~nm})$. 


\section{Supplementary characterization, table and figures}
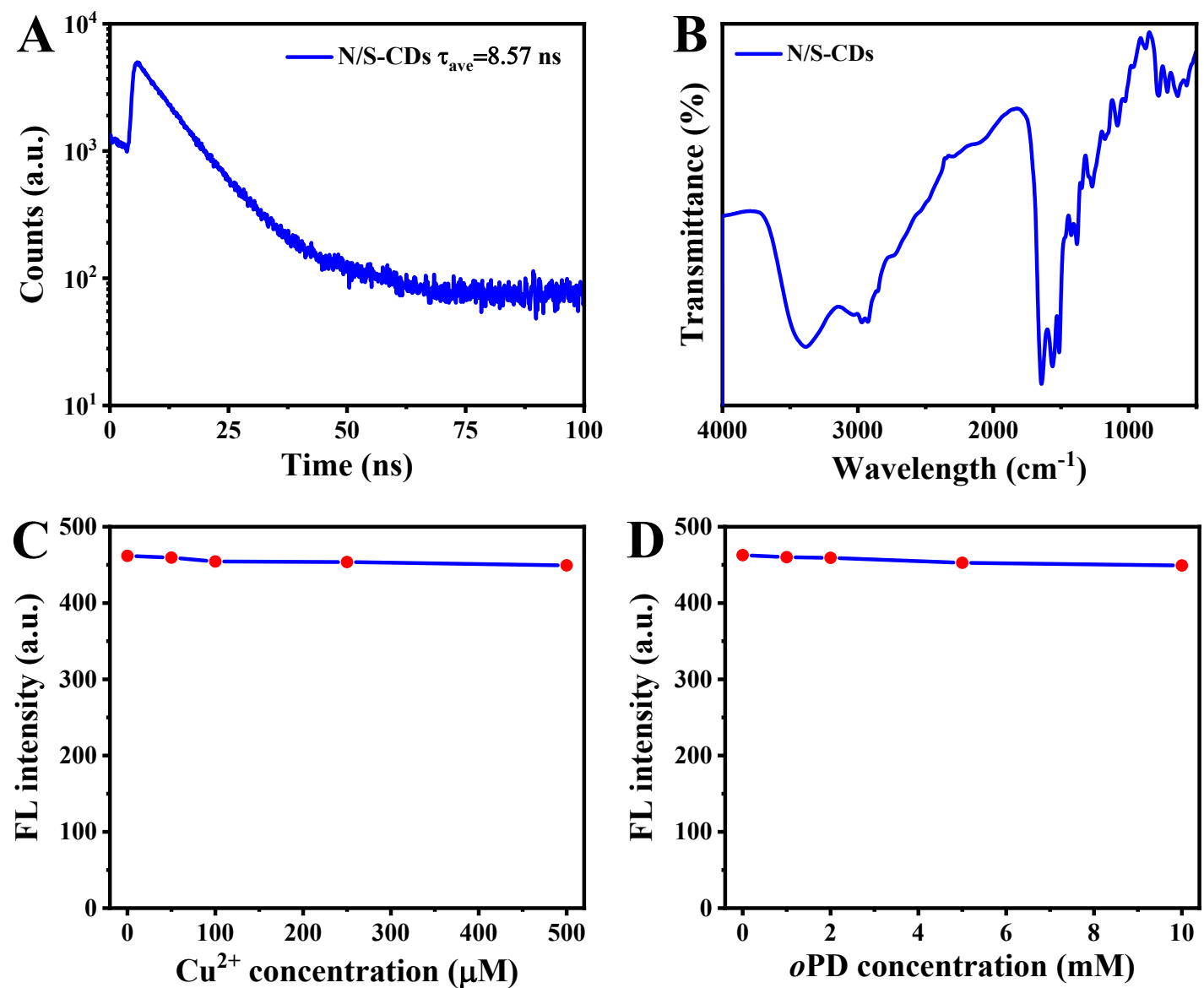

Figure S1. (A) Transient fluorescence spectra of N/S-CDs. (B) FT-IR spectrum of N/S-CDs. Effects of different concentrations of $\mathrm{Cu}^{2+}(\mathrm{C})$ and $o \mathrm{PD}$ (D) on the fluorescence intensity of N/S-CDs. 

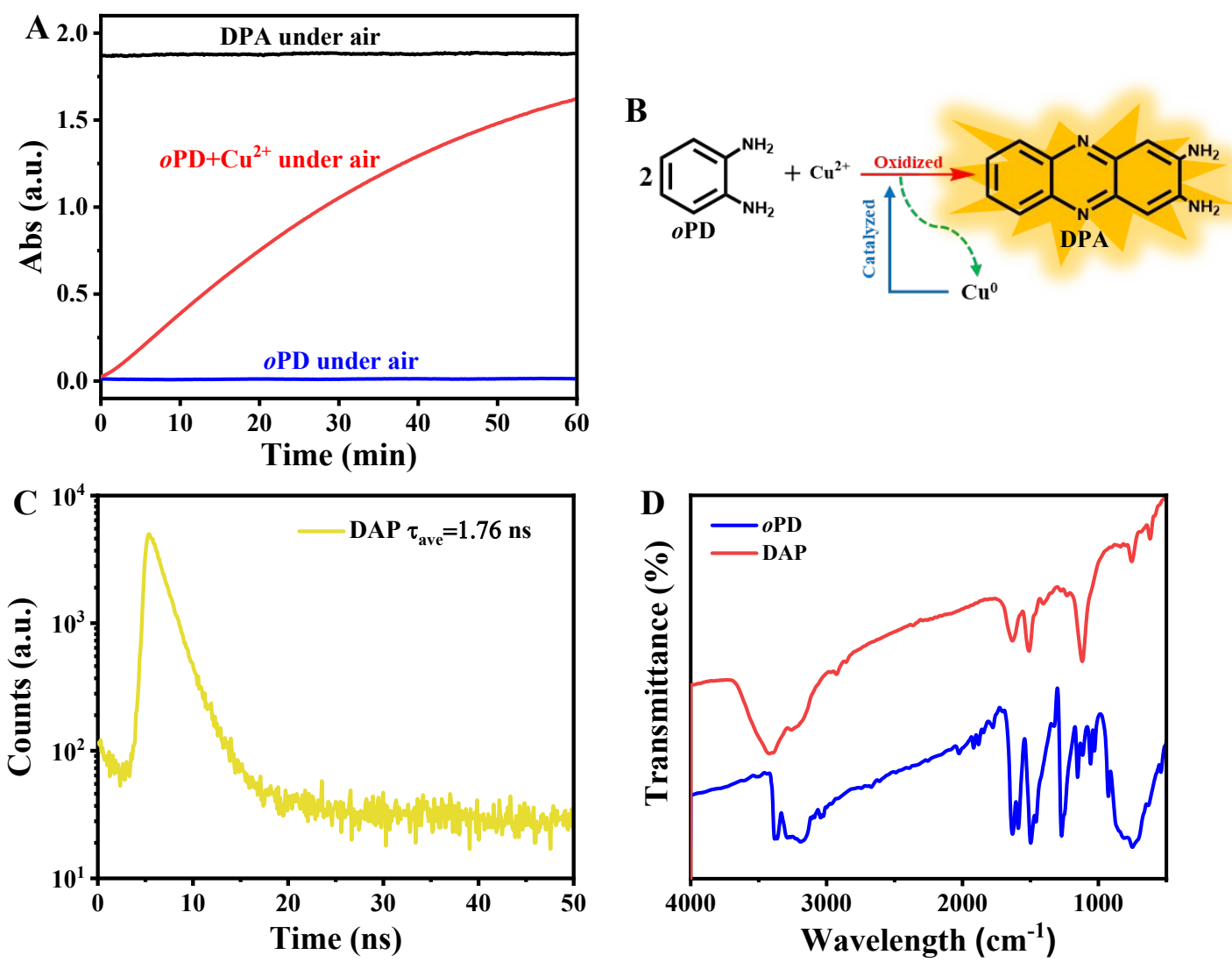

Figure S2. (A) The change of absorbance value with time during the reaction of $o \mathrm{PD}$ and $\mathrm{Cu}^{2+}$. (B) Schematic diagram of $\mathrm{Cu}^{2+}$ oxidizes $o$ PD to DAP. (B) Transient fluorescence spectra of DAP. (C) FT-IR spectra of $o$ PD and DAP.

We use freshly prepared $o \mathrm{PD}$ and $\mathrm{CuCl}_{2}$ solutions to investigate the kinetic relationship of product absorbance over time, and DPA is a purchased commercial reagent (Shanghai Aladdin Reagent, China). As shown in the Figure S2A, $o$ PD alone $(50 \mu \mathrm{L}, 20 \mathrm{mM})$ is hardly oxidized by oxygen in the air within $60 \mathrm{~min}$, while the mixed solution of $o \mathrm{PD}(50 \mu \mathrm{L}, 20 \mathrm{mM})$ and $\mathrm{Cu}^{2+}(20 \mu \mathrm{L}$, 4.0 $\mathrm{mM}$ ) reacts quickly to form DPA in the air, and the same DPA hardly changes in the air. This result indicates that the formation of DPA by $o$ PD in this work requires the participation of $\mathrm{Cu}^{2+}$. Besides, the molecular ratio of $\mathrm{Cu}^{2+}$ to $o \mathrm{PD}$ is 2:25, It is speculated that $o \mathrm{PD}$ is not only oxidized by $\mathrm{Cu}^{2+}$, but also oxidized by oxygen in the air under the catalyzing of the reduction product of $\mathrm{Cu}^{2+}$ (e.g. CuNPs) (Figure S2B). 


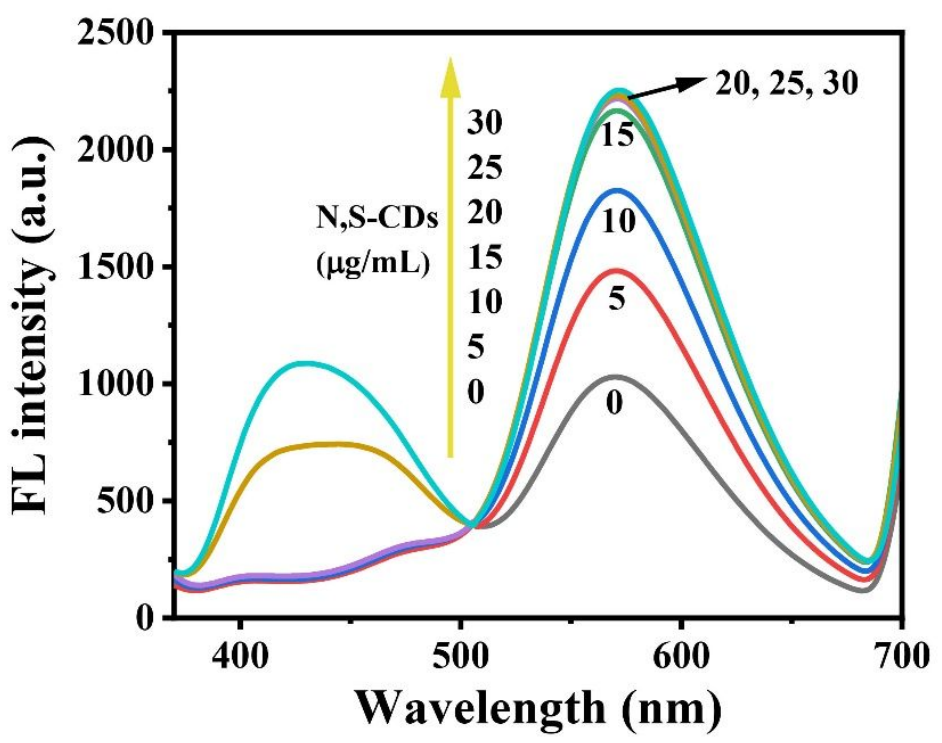

Figure S3. Concentration of N/S-CDs in FRET ratiometric fluorescence system. 


\section{Analysis of FRET efficiency:}

(1) The transfer efficiency is typically measured using the relative fluorescence intensity of the donor, in the absence $\left(F_{\mathrm{D}}\right)$ and presence $\left(F_{\mathrm{DA}}\right)$ of acceptor (Figure $\left.3 \mathrm{~A}\right)$ :

$$
E=1-\frac{F_{D A}}{F_{D}}=1-\frac{159.1}{2341}=0.932
$$

(2) The FRET efficiency was calculated based on the distance when the D-A distance is near $R_{0}$.

The detailed calculation steps are as follows:

$R_{0}$ is the Förster distance, and $r$ is the donor-toacceptor distance.The Förster radius $\left(R_{0}\right)$ for which the energy transfer efficiency is diminished to $50 \%$ of the maximal value is calculated using eqn (S2).

$R_{0}^{6}=\frac{9000(\ln 10) \kappa^{2} Q_{D}}{128 \pi^{5} N n^{4}} \int_{0}^{\infty} F_{D}(\lambda) \varepsilon_{A}(\lambda) \lambda^{4} d \lambda=8.96 \times 10^{-5}\left(\kappa^{2} n^{-4} Q_{D} J\right) \quad\left(\right.$ in $\left.\AA^{6}\right)$

where $k^{2}$ is an orientation factor, which is regarded as $2 / 3$ for a randomly orientated donoracceptor pair; $n$ is the refractive index of the medium, which is 1.33 for water; $Q_{D}$ is the quantum yield of the donor in the absence of acceptors, which is 0.72 for N,S-CDs; $J$ is the spectral integral as a function of wavelength, expressing the spectral overlap between the emission spectrum of the donor and the absorption spectrum of the acceptor, which is obtained by eqn (S3)

$$
J=\frac{\int_{0}^{\infty} F_{D}(\lambda) \varepsilon_{A}(\lambda) \lambda^{4} d \lambda}{\int_{0}^{\infty} F_{D}(\lambda) d \lambda} \quad\left(\mathrm{M}^{-1} \mathrm{~cm}^{-1} \mathrm{~nm}^{4}\right)
$$

Where $F_{D}(\lambda)$ is the dimensionless emission intensity; $\lambda$ is the wavelength; $\varepsilon_{A}(\lambda)$ is the molar absorption coefficient of DAP at $\lambda$. The calculated overlap integral $J$ is around $2.1645 \times 10^{14} \mathrm{M}^{-1} \mathrm{~cm}^{-}$ ${ }^{1} \mathrm{~nm}^{4}$. Thus the Förster radius $R_{0}$ is calculated to be $37.92 \AA$ according to eqn (S2) .

The efficiency $E$ of a FRET process can also be calculated using eqn (S4):

$$
E=\frac{R_{0}^{6}}{R_{0}^{6}+r^{6}}
$$




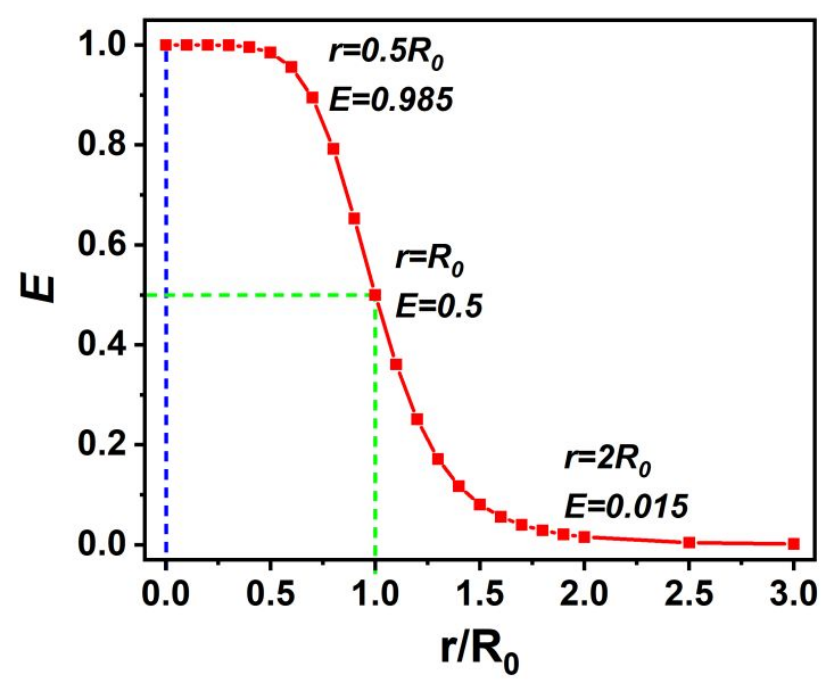

Figure S4. Dependence of the energy transfer efficiency $(E)$ on distance. $R_{0}$ is the Förster distance, and $r$ is the donor-toacceptor distance.

In summary, we have given the above two methods for the calculation of FRET efficiency, and the FRET efficiency of the relative method is $93.2 \%$. Another method shows that the transfer efficiency is strongly dependent on distance when the $D-A$ distance is near $R_{0}$ (Figure S4). However, the donor-toacceptor distance $(r)$ cannot be calculated, so we cannot calculate $E$ by a correction method. But we calculated $J$ by measuring the parameters to be $2.1645 \times 10^{14} \mathrm{M}^{-1} \mathrm{~cm}^{-1} \mathrm{~nm}^{4}$ and the Förster radius $\left(R_{0}\right)$ is $37.92 \AA$. 
Table S1. Comparison of the FRET efficiency of different FRET sensing or imaging platforms.

\begin{tabular}{cccc}
\hline $\begin{array}{c}\text { FRET Mode } \\
\text { (Donor@Acceptor) }\end{array}$ & $\begin{array}{c}\text { FRET } \\
\text { Efficiency }\end{array}$ & Application & Reference \\
\hline CD@MOF & $68.7 \%$ & Hypochlorous Acid & Anal. Chem. 2020, 92, 3447-3454. \\
\hline CdSe/ZnS@Dye & $78.5 \%$ & Neutrophil Elastase & ACS Nano 2020, 14, 4244-4254. \\
\hline NP@ATTO665 & $51 \%$ & Oligonucleotides & Angew. Chem. Int. Ed. 2020, 59, 6811- \\
\hline UCNP@fluorescent & $20 \%$ & 6818. & \\
\hline protein & & pH & Biosens. Bioelectron. 2020, 155, 112115. \\
\hline $\begin{array}{c}\text { Iridium(III) } \\
\text { complex@RhB }\end{array}$ & $26 \%$ & Caspase-3 & J. Am. Chem. Soc. 2020, 142, 1057-1064. \\
\hline mTagBFP2@FAD & $40 \%$ & Optogenetics & Nat. Methods 2019, 16, 1029-1036. \\
\hline CDs@DAP & $93.2 \%$ & ALP & This work \\
\hline
\end{tabular}


Table S2. Comparison of the sensing performance of different methods for detecting ALP activity.

\begin{tabular}{cccc}
\hline Method & Linear range (U/L) & LOD (U/L) & Reference \\
\hline SERS & $0.5-10$ & 0.1 & Sensor. Actuat. B-Chem. 2017, 253, 839-845 \\
\hline Electrochemical & $20-1500$ & 3.0 & Sensor. Actuat. B-Chem. 2019, 281, 221-228 \\
\hline $\begin{array}{c}\text { Colorimetric } \\
\text { Chemiluminescence }\end{array}$ & $5-100$ & 3.3 & ACS Appl. Mater. Inter. 2014, 6, 18243- \\
\hline Fluorescence & $0-30$ & 1.0 & Luminescence 2017, 32, 1150-1156 \\
\hline Fluorescence & $3.4-100$ & 0.9 & Biosens. Bioelectron. 2016, 83, 274-280 \\
\hline
\end{tabular}

